Gut, 1978, 19, 593-601

\title{
Quantitative comparison of the effects of cholecystokinin, secretin, and pentagastrin on gastrointestinal myoelectric activity in the conscious fasted $\operatorname{dog}^{1}$
}

\author{
D. L. WINGATE, ${ }^{2}$, E. A. PEARCE, M. HUTTON, A. DAND, \\ H. H. THOMPSON, AND E. WÜNSCH
}

From the Gastroenterology Unit and Departments of Experimental Surgery and Physiology, London Hospital Medical College, London, and the Max Planck Institute of Biochemistry, Munich, West Germany

SUMMARY The effects on gastrointestinal myoelectric activity of infused pentagastrin, cholecystokinin (CCK), and secretin at physiological doses were studied in live dogs with implanted serosal electrodes during 56 six-hour studies. Pentagastrin dose-dependently increased gastric and duodenal slow-wave frequencies; secretin and CCK did not. Pentagastrin and CCK diminished the incidence of fasting migrating myoelectric complexes (MMCs), but MMCs were abolished only in the proximal small intestine. Pentagastrin infusion was not reflected in an increased number of spikes, whereas CCK induced a dose-dependent increase in jejunal spike activity. Secretin dose-dependently decreased duodenal and jejunal spike incidence without a marked effect on MMC incidence. Analysis of patterns of spike activity showed significant dose-dependent changes with all three peptides. The different effects of pentagastrin and CCK on spike activity in these studies may have been a consequence of pentagastrin-stimulated gastric acid secretion. None of the three peptides produced a pattern of myoelectric activity which closely resembled that seen on feeding; since, unlike food, all three peptides had little or no effect on the distal small intestine, it seems unlikely that combinations of these peptides are responsible for the change induced by food. The failure of these peptides to abolish fasting patterns in the distal intestine suggests a possible mechanism for some types of postvagotomy dysfunction.

Pentagastrin has been shown to simulate the effects of feeding on myoelectric activity (Weisbrodt et al., 1974; Marik and Code, 1975), and a similar effect of cholecystokinin (CCK) has been reported recently (Mukhopadhyay et al., 1977). Such studies have raised the possibility that these peptides may be the agents responsible for the change from interdigestive to digestive myoelectric activity, but the measurement required to resolve the question was not then

\footnotetext{
${ }^{1} \mathrm{~A}$ preliminary account of part of this work was presented to the 6th International Symposium on Gastrointestinal Motility in Edinburgh (Wingate, Thompson, Pearce, and Dand, 1978).

${ }^{2}$ Address for reprint requests: $D$. L. Wingate, London Hospital Medical College, London E1 1BB, England.
}

Received for publication 29 March 1978 available. Secretin has not been studied in this context, but one study (Hermon Taylor and Code, 1970) suggests that it inhibits myoelectricactivity. New methods of recording and analysing myoelectric activity (Wingate, Barnett, et al., 1977; Wingate and Barnett, 1978) which allow the incidence and patterns of spike activity to be measured have been used in this study to analyse the myoelectric response to the three hormones, CCK, secretin, and gastrin; for the latter two substances pure peptides were used. The new methodology has not merely simplified quantitative study but has also greatly reduced the time required to analyse the data. This has permitted study of the effects of these peptides over a physiological dose range; the results presented in this paper are derived from 56 experiments, each lasting six hours 
and analysed with a numerical and graphic record in approximately 30 minutes.

\section{Methods}

\section{ANIMALS}

The subjects were five adult Labrador dogs (weight $25-27 \mathrm{~kg}$ ) in whom an array of implanted monopolar electrodes had been sutured to the serosa of the digestive tract between the gastric antrum and the terminal ileum, up to six months before study. The electrode leads terminated in a cannula in the abdominal wall of the animal incorporating an electrical connector for connection to an external recording device. The metal cannula also served as an indifferent electrode. Before surgical implantation of the electrodes the animals had been trained to stand for long periods of time supported by a sling.

\section{RECORDING TECHNIQUES}

Tape

Myoelectric activity was continuously recorded on Phillips $\mathrm{C} 120 \frac{1}{8}$ in tape using a Medilog 4-24 cassette tape-recorder (Oxford Electronic Instruments, Abingdon, Oxon.) from three sites in the animals. These sites were the distal duodenum, mid-jejunum, and terminal ileum. Spike activity was analysed on replay (Wingate, Barnett. et al., 1977). During recording the recorded signals were monitored directly from the tape head on an oscilloscope.

\section{Direct}

For analysis of the basic electrical rhythm samples of electrical activity were recorded from the gastric antrum and from the duodenum using a fibreoptic recording oscilloscope to produce a direct graphic record.

\section{EXPERIMENTAL SUBSTANCES}

The test substances used were pentagastrin (Peptavlon, ICI), highly purified cholecystokinin (GIH Research Laboratories, Karolinska Institute, Stockholm), and pure synthetic secretin. Doses were within the physiological range, and are shown in the Table. All substances were administered by intravenous cannula using a constant infusion syringe pump; peptides were diluted to give an infusion rate of $24 \mathrm{ml} / \mathrm{h}$.

The preparation of CCK used in these studies is calibrated in Ivy dog units. Conventionally, one Ivy dog unit of CCK is assumed to be equivalent to 4 Crick-Harper-Raper units of CCK; Boots CCK (Boots Company Ltd., Nottingham, England) is calibrated in the latter units. It has recently been shown (Oliver and Harvey, 1977) that the generally accepted relative potencies of the two preparations
Table Doses of test substances

\begin{tabular}{|c|c|c|}
\hline \multicolumn{2}{|l|}{ Infusion dose $(U / \mathrm{kg} / \mathrm{h})$} & Dogs \\
\hline \multicolumn{3}{|l|}{ Cholecystokinin } \\
\hline \multicolumn{2}{|l|}{0.125} & A, C, L $\mathbf{H}$ \\
\hline \multicolumn{2}{|l|}{0.25} & A, C, L H \\
\hline \multirow{2}{*}{\multicolumn{2}{|c|}{$\begin{array}{l}0.5 \\
1.0\end{array}$}} & A, C, L H \\
\hline & & A, C, L, H \\
\hline \multicolumn{2}{|l|}{2.0} & A, C, $\vec{L}, \mathbf{H}$ \\
\hline \multicolumn{3}{|l|}{ Pentagastrin } \\
\hline \multicolumn{2}{|l|}{$0 \cdot 125$} & $\mathbf{A}, \mathbf{C}, \mathbf{L}, \mathrm{H}$ \\
\hline \multicolumn{2}{|l|}{0.25} & A, C, L, H \\
\hline \multicolumn{2}{|l|}{0.5} & A, C, L, H \\
\hline \multicolumn{2}{|l|}{1.0} & A, C, L, H \\
\hline \multicolumn{2}{|l|}{$2 \cdot 0$} & A, C, L, H \\
\hline 4.0 & & A, C, L, H \\
\hline \multicolumn{3}{|l|}{ Secretin } \\
\hline \multicolumn{2}{|l|}{0.5} & C, $\mathbf{L}, \mathbf{R}$ \\
\hline \multicolumn{2}{|l|}{$1 \cdot 0$} & C, $\mathbf{L}, \mathbf{R}$ \\
\hline \multirow{2}{*}{\multicolumn{2}{|c|}{$\begin{array}{l}2.0 \\
3.0\end{array}$}} & A, C, R \\
\hline & & A, C, H \\
\hline \multirow{3}{*}{\multicolumn{3}{|c|}{ 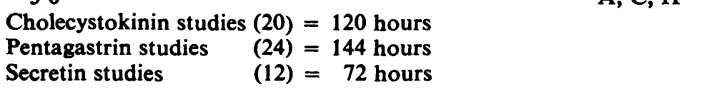 }} \\
\hline & & \\
\hline & & \\
\hline \multicolumn{3}{|c|}{ dies $=336$ ho } \\
\hline
\end{tabular}

may be misleading, since, on bioassay, they found one nominal Crick-Harper-Raper unit of Boots CCK to be approximately twice as potent as one nominal Ivy dog unit of Karolinska Institute CCK. According to the radioimmunoassay of Oliver and Harvey (1977), Boots CCK is highly potent compared to Karolinska CCK, but much of the immunoreactivity consists of short peptide chains which are not bioactive-that is, not CCK. Some indication of the 'physiological' dose of CCK has been provided by studies by Harvey et al., (1977) who showed that the postprandial level of CCK in man is equivalent to the level produced by an infusion rate of CCK at 2 Ivy dog units $/ \mathrm{kg} / \mathrm{h}$. This is the highest infusion rate used in these studies.

The synthetic secretin used in these studies is supplied in microgram quantities. Because the dosage of secretin is generally expressed in units, and this forms the basis for comparison with other studies of this peptide, our infusion rates of secretin were calculated in units, using the conversion factor of $3.5 \mathrm{U} / \mathrm{micro}-$ gram obtained for this preparation of synthetic secretin against pure secretin (Mutt) and secretin with cysteine (GIH Research Laboratory) in studies of canine pancreatic secretion (Lehnert et al., 1973).

\section{PROTOCOL}

Each animal was fasted for 18 hours before study, and each study lasted for six hours. Studies in which characteristic fasting activity was not initially present were rejected and the experiment was repeated. The first two hours of each study was a control record of fasting myoelectric activity; during this time a slow 
$(24 \mathrm{ml} / \mathrm{h}$ ) saline infusion was administered. At the end of two hours, the peptide infusion replaced the saline infusion and the peptide was administered for a further two hours. At the conclusion of the peptide infusion, the saline infusion was resumed and the recording continued for two more hours, making a total of three two-hour periods for each study. The beginning and end of each of the three test periods in each study was marked on the tape by an electric signal which was used during replay in the analysis of data. Thus the standard experiment, as illustrated in Fig. 1, comprised a control period, a test period with peptide infusion, and a further control period in which the rapidity of recovery from the peptide could be assessed. The total number and duration of studies of the three peptides are listed in the Table.

\section{DATA ANALYSIS}

\section{Basic electrical rhythm (BER)}

BER was calculated from samples of recorded activity taken at 30 minute intervals during each study. Each sample consisted of a five minute period of recording and slow waves on the recording were counted for this period to yield an average frequency. For the purposes of comparison, values taken from the middle of the control and test periods were used.

\section{Spike activity}

Spike activity was analysed on high speed replay (Wingate et al., 1977). During replay each channel of recorded activity was replayed at high speed and during the replay recorded spikes were isolated by active filtering and converted into digital pulses. These digital pulses were used in three ways. First, they were used to construct a histogram of spike activity in which the incidence of migrating myoelectric complexescould beclearly detected. Secondly, total spike activity for each period during the study at each recording site was derived from spike totals displayed on digital counters, which were latched and reset by the event pulses recorded during the study. Finally, the incidence of patterns of spike activity was calculated using an automatic analyser (Wingate and Barnett, 1978). The analyser discriminates between three types of spike activity (Barnett and Wingate, 1977)-absent spike activity, intermittent spike activity and activity fronts, type 1,2, and 3, respectively - which correspond roughly to phase 1 , 2 , and 3 activity as described by Code and Marlett (1975). The analyser records both the period of time during the study period occupied by one or other of the types of activity, and also the total number of spikes contributing to either type 2 or type 3 activity. For example, the analyser can discriminate between a recording in which there is no change in the time occupied by intermittent spiking activity but there is an absolute increase in spikes, and a recording in which the time occupied by intermittent spike activity is greatly increased, without a concomitant increase in the total number of recorded spikes.

\section{Statistical analysis}

Since each study included both a control and a test period, changes between these two periods were compared using a paired $t$ test to determine the probability (P) of significant change. Estimations of dose-dependency were made by calculating the linear regression of data points at different peptide con-

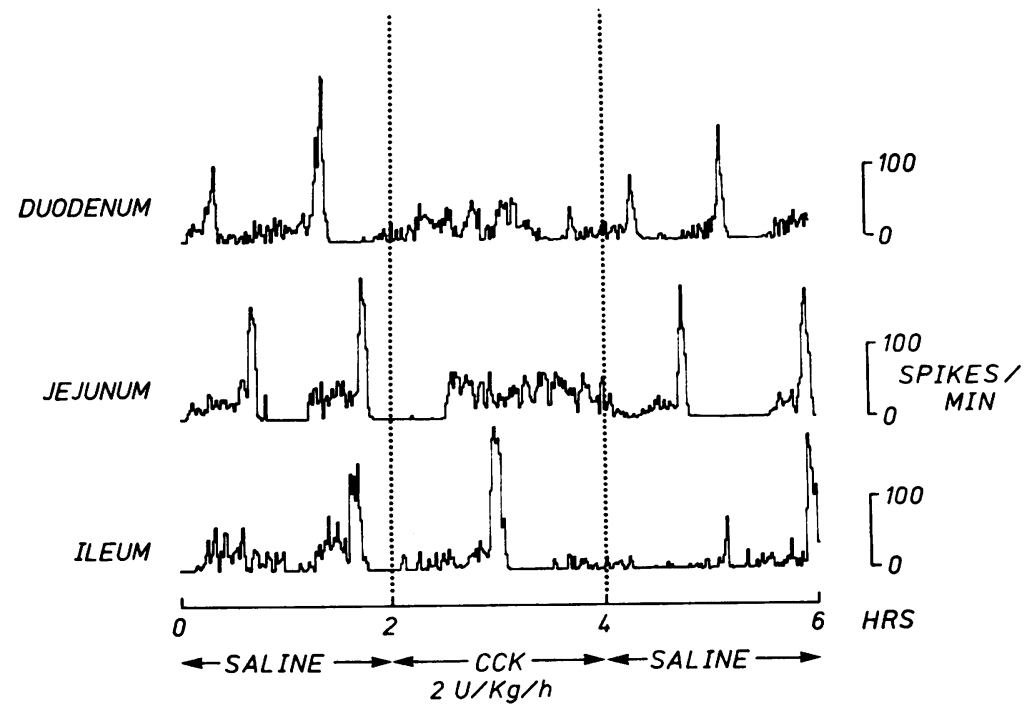

Fig. 1 Histogram of spike activity from a single study with CCK infusion, illustrating the standard experimental design. This study, showing virtually unimpaired fasting spike activity in the ileum during the peptide infusion, was typical of many of the studies with CCK or pentagastrin infusion. 
centrations against the logarithmic peptide concentration. In the pentagastrin and CCK studies, where the same four animals were used for all peptide concentrations, calculation of regressions of dose response for each animal might have been used. We have chosen the regression calculations for the grouped data to simplify the illustration of results to a single regression line with mean values as individual data points.

\section{Results}

\section{BASIC ELECTRICAL RHYTHM (BER)}

Pentagastrin was clearly differentiated from the other two peptides in its effect on the BER frequency. Pentagastrin induced a dose-dependent increase in both gastric and duodenal BER frequencies, while CCK had no accelerating effect on either (Fig. 2). At the lowest dose $(0 \cdot 125 \mathrm{U} / \mathrm{kg} / \mathrm{h})$ of CCK, there was an apparent fall in BER from fasting values $(P<0.05)$,
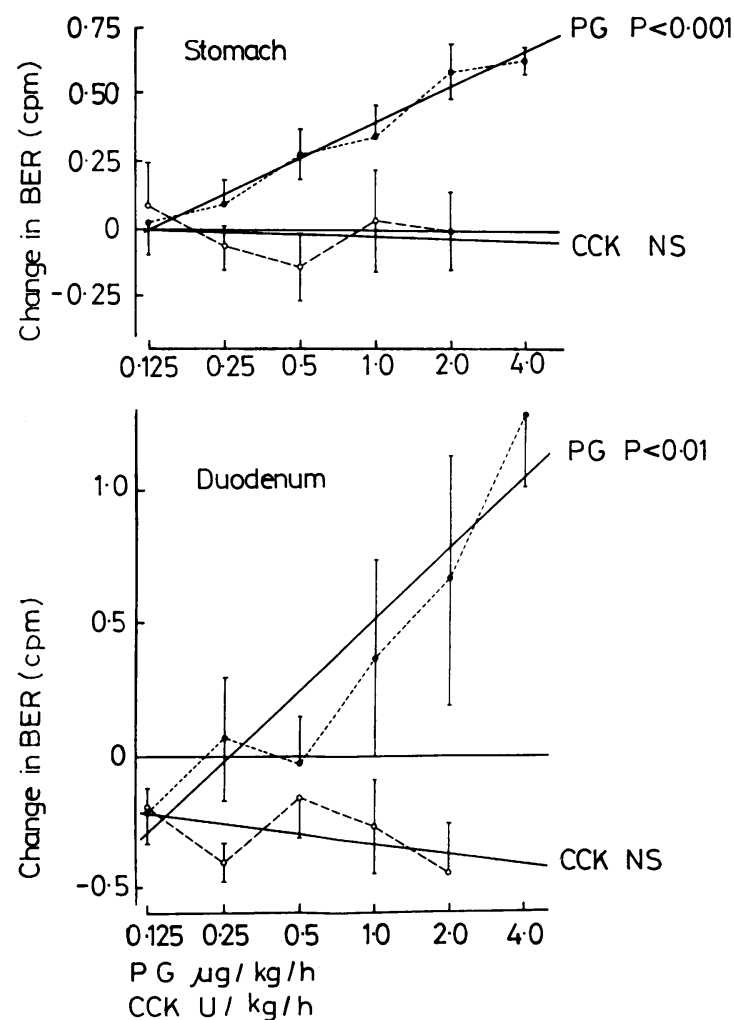

Fig. 2 The effect of $C C K(\bigcirc)$ and pentagastrin (O) on the basic electrical rhythm of the stomach (above) and the duodenum (below). Each point represents the mean of four studies. Straight lines show the calculated linear regressions for frequency change/log dose of pentagastrin (PG) or cholecystokinin (CCK). and a similar change in duodenal BER was seen with the lowest dose of secretin (Fig. 3); again, this was just statistically significant. Both duodenal and gastric BERs showed an apparent dose-dependent increase with increasing secretin dosage (Fig. 3), but the data are also compatible with an alternative explanation: it is possible that low doses of secretin reduce the BER, while higher doses restore normal values. While the functional and physiological significance of such changes remains in question, alternative interpretations cannot be discarded.

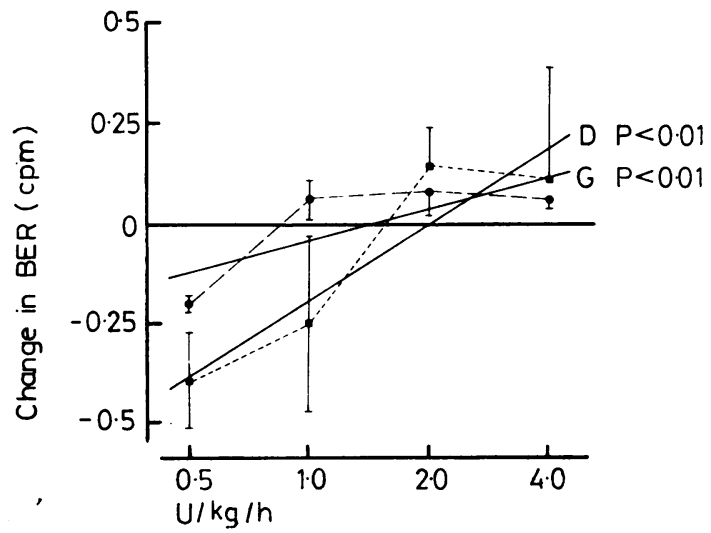

Fig. 3 Each point represents the mean ( \pm 1 SEM) of three observations of change in gastric $(\bullet)$ or duodenal $(\square)$ $B E R$ frequency change. Straight lines show the calculated linear regressions for change in gastric $B E R(G)$ and duodenal BER $(D)$ against log secretin dose.

\section{MIGRATING MYOELECTRIC COMPLEXES}

(MMC)

The effect of the peptides on the incidence of MMCs is illustrated in Figs 4-6. Pentagastrin and CCK had similar effects on the proximal small intestine, but relatively little effect on the ileum. Pentagastrin (Fig. 4) appeared to diminish MMC incidence in both duodenum and jejunum at all but the lowest infusion dose, but complete abolition of duodenal MMCs was achieved only by the two highest doses. Ileal MMCs were but little affected by pentagastrin. The two lowest doses of CCK were without effect (Fig. 5), but the greatest inhibitory effect was seen with CCK infusion at $0.5 \mathrm{U} / \mathrm{kg} / \mathrm{h}$ with virtually complete abolition of duodenal and jejunal MMCs. CCK, like pentagastrin, induced only a moderate reduction in ileal MMCs. The smaller number of studies with secretin have produced somewhat scattered data (Fig. 6), but the only effect of secretin appears to be inhibition of jejunal MMCs at the two higher infusion doses.

TOTAL SPIKE ACTIVITY

The effects of CCK and pentagastrin on total spike 

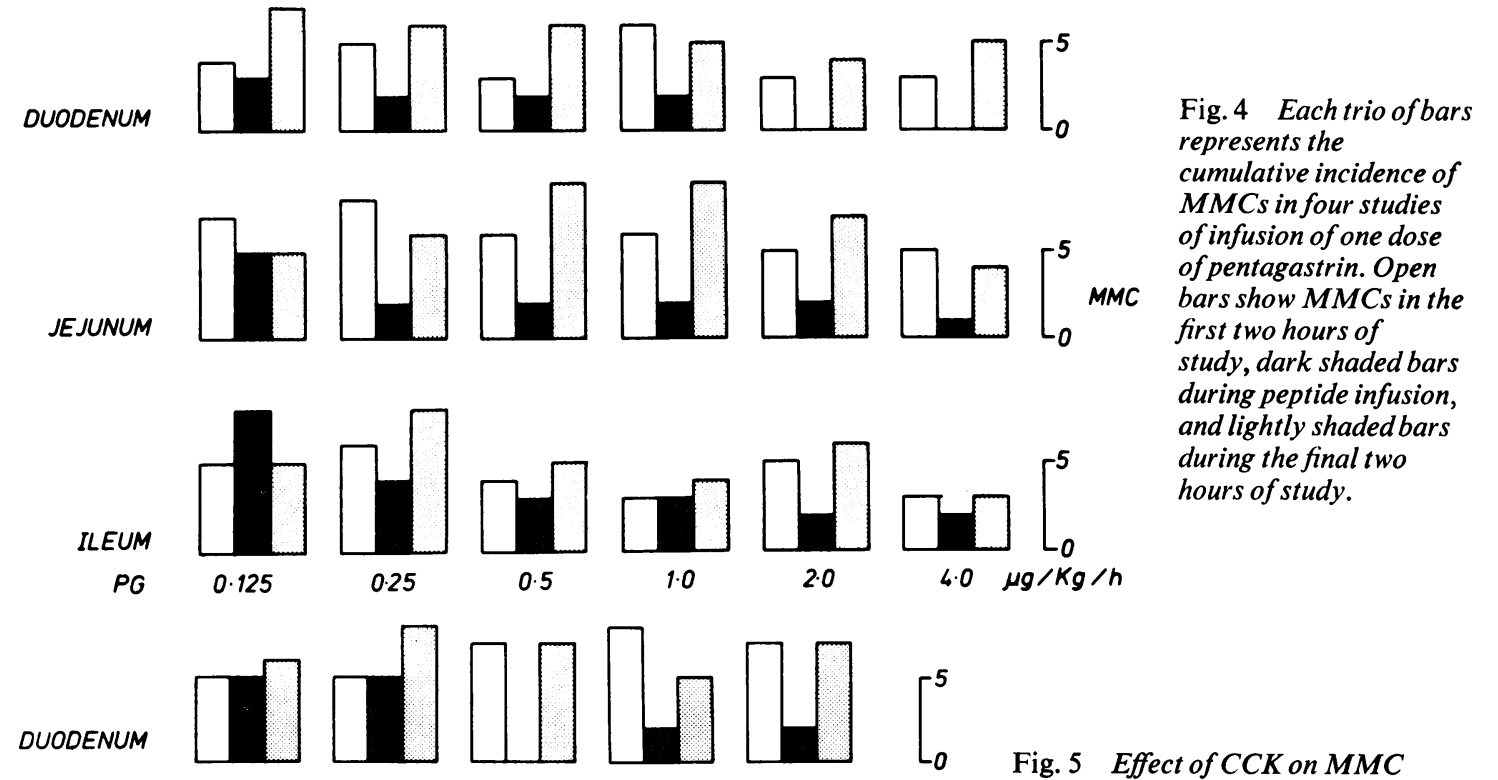

Fig. 5 Effect of CCK on MMC

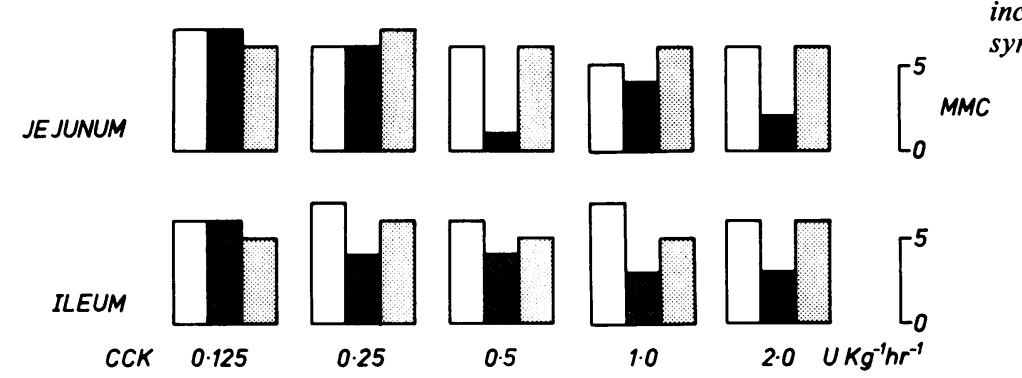
incidence. For explanation of symbols see Fig. 4.
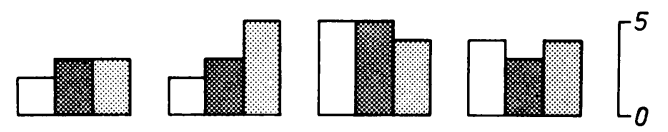

JEJUNUM
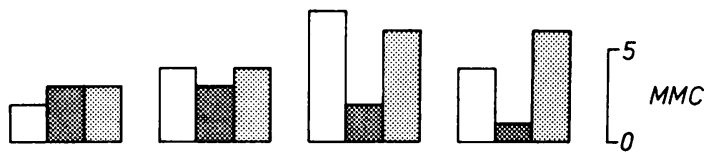

Fig. 6 Effect of synthetic secretion on MMC incidence. For explanation of symbols see Fig. 4; note that each trio of bars represents cumulative incidence in three (not four) studies.

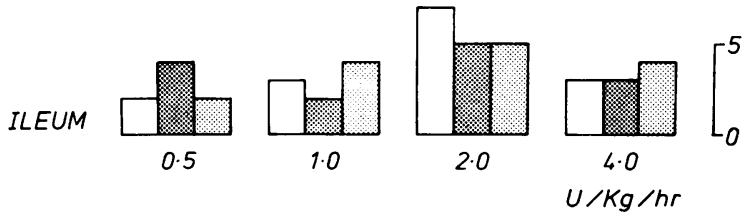

activity calculated by expressing the number of spikes during peptide infusion as a percentage of the number of spikes during the preceding control period, was less than expected. Indeed, pentagastrin (Fig. 7) had no effect on overall spike incidence over the entire dose range. In contrast, CCK (Fig. 7) induced a significant $(P<0.001)$ dose-dependent increase in jejunal spike activity, but was without effect on ileal and duodenal spike activity. At the highest infusion dose of CCK, the increase in spike 


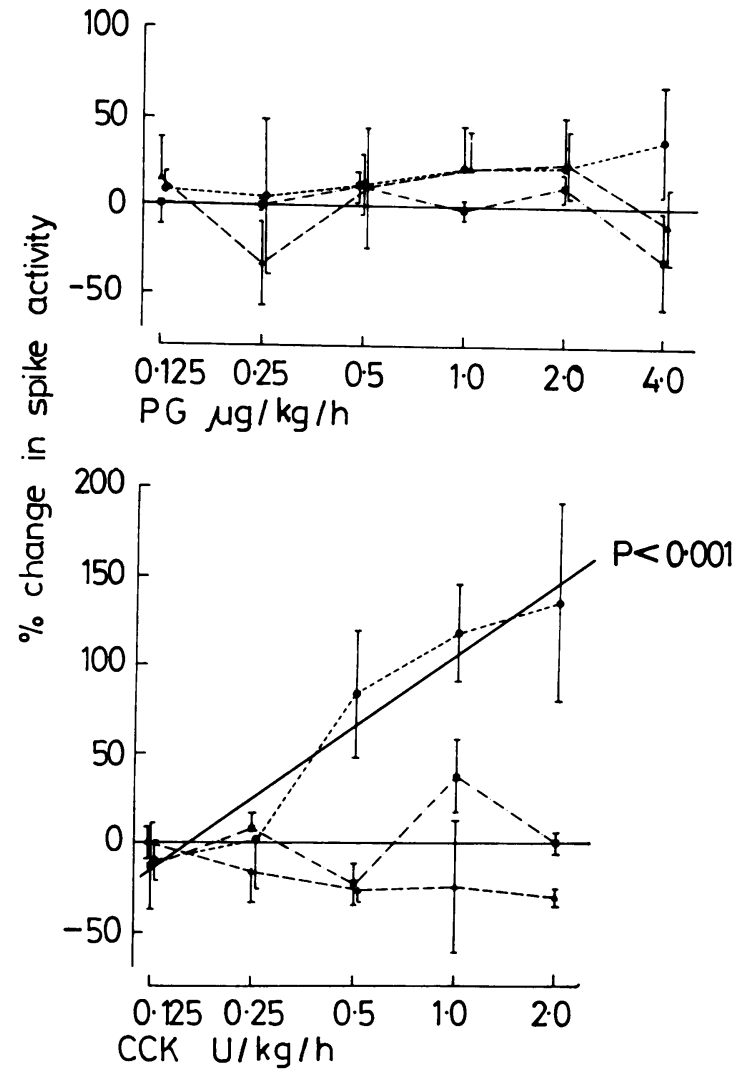

Fig. 7 Change in total spike activity between control periods and periods of pentagastrin infusion (above) and CCK infusion (below). The broken lines connect mean data points; the straight line in the lower panel is the calculated linear regression of change in jejunal spike activity with CCK log concentration. - - D Duodenum. - - - Jejunum. - $\triangle$ Ileum.

activity was in the order of $50 \%$. Secretin (Fig. 8) had a clear inhibitory effect on spike activity in the proximal and mid-bowel, which was in the order of $75 \%$ inhibition at the highest dose. Because of scatter in the data, the inhibition of ileal spike activity was not statistically significant.

\section{PATTERNS OF SPIKE ACTIVITY}

Analysis of changes in patterns of spike activity in general followed the changes previously described. There was no statistically significant change in type 3 (repetitive spike bursts) activity with the peptides. Both CCK and pentagastrin dose-dependently increased type 2 (intermittent spike activity) activity in the jejunum, and CCK had a similar effect on the duodenum (Fig. 9). Since either type 1 or type 2 activity is present for most of any given period, it follows that this increase in type 2 activity must have

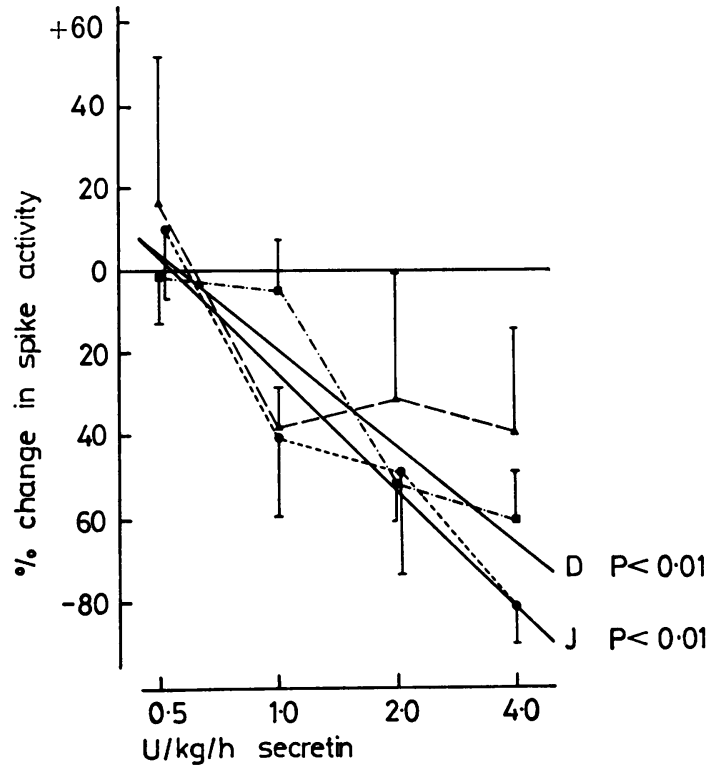

Fig. 8. Change in total spike activity between control periods and periods of secretin infusion. Linear regressions, shown as straight lines for duodenal activity $(D)$ and jejunal activity $(J)$, were calculated from mean change against secretin log concentration. - - D Duodenum (mean $\pm 1 S E M)$. ---- Jejunum (mean $\pm 1 S E M)$. $-\triangle$ Ileum (mean \pm 1 SEM).

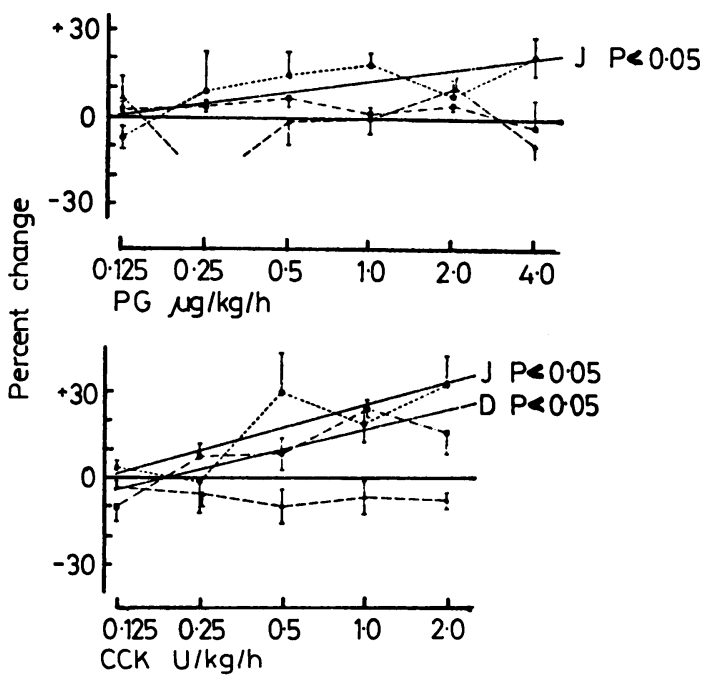

Fig. 9 Change in incidence of type 2 (intermittent spike) activity between control periods and periods of pentagastrin (above) and CCK (below) infusion. Broken lines connect mean values $(n=4) \pm 1 S E$; straight lines show calculated linear regressions $(\%$ change vs. peptide log dose) for jejunal activity $(J)$ and duodenal activity $(D)$. - - - Duodenum. - - - - Jejunum. - $\triangle$ Ileum. 
been at the expense of type 1 (absent spike activity) activity, and analysis confirmed a reciprocal fall in type 1 activity for these two peptides of equal statistical significance $(P<0.05)$. Secretin induced an increase in the duration of type 1 activity (Fig. 10) in the duodenum and ileum, but a similar trend in the jejunum did not achieve statistical significance; again this increase in type 1 activity was accompanied by a reciprocal fall in type 2 activity of equal significance.

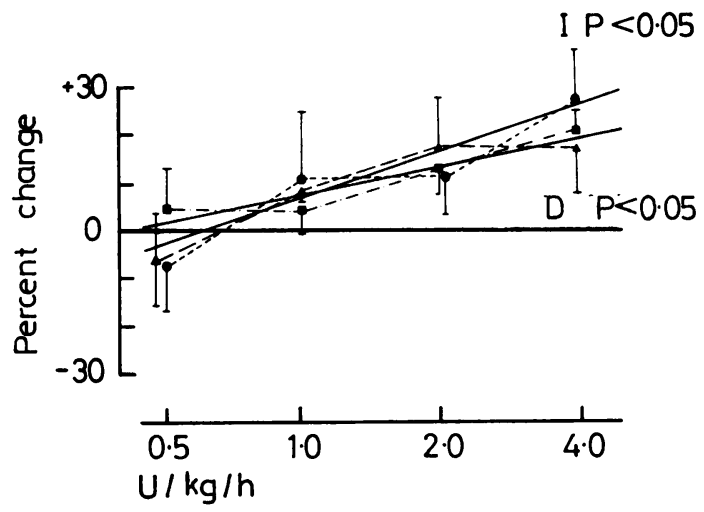

Fig. 10 Change in duration of type 1 activity between control periods and periods of secretin infusion. Broken lines connect mean values $(n=3) \pm 1$ $S E$. Straight lines show calculated linear regressions ( $\%$ change vs. secretin log dose) for duodenum $(D)$ and ileum $(I)$. - - D Duodenum (mean \pm 1 SEM). - Jejunum (mean \pm 1 SEM $).-\triangle$ Ileum $($ mean $\pm 1 S E M)$.

\section{Discussion}

\section{INTERPRETATION OF RESULTS}

The results presented in the preceding section do not, at first sight, fall into a coherent pattern; all three peptides exhibit both stimulant and inhibitory effects on myoelectric activity. They are more easily understood in terms of a model of myoelectric activity which includes both the ability of intestinal muscle to vary spike activity, and the existence of an extraenteric clock which controls cyclical fasting activity (Wingate, 1976). The experimental basis of this model is the constancy of cyclical fasting activity and its disruption by feeding (Code and Marlett, 1975) and the demonstration of extra-enteric control (Carlson et al., 1972) which is not dependent upon intact vagi (Marik and Code, 1976). During fasting, spike activity in the small intestine is grouped into recurrent cycles of absent spike activity, intermittent spike activity, and intense repetitive spike bursts, which were called phase I, II and III respectively by Code and Marlett (1975). In an average cycle of 100 minutes, phase III occupies less than five minutes and phases I and II, of roughly equal duration, occupy the rest of the time. According to our model, during fasting, when chyme is absent, it is the extra-enteric 'clock' which dictates the spike activity of the intestine; on feeding, 'clock' control is disconnected and spike activity is then dictated by circulating humoral factors (endocrine) and local responses to luminal stimuli (neural and paracrine).

According to this model, myoelectric spike activity may be modified in two principal ways. The 'clock' control may be disconnected, abolishing cyclical activity; in this case it may be expected that spike activity will show a relatively dose-dependent response to humoral stimulus. Alternatively, the clock control may remain but the myoelectric complex itself may be modified in terms of duration of phase I, II, and III.

Against this background, our results can be interpreted in the following manner. Both CCK and pentagastrin tend to disconnect 'clock' control by abolition of cyclical spike activity (MMCs) in the duodenumand jejunum. In the jejunum, CCK induces a dose-dependent increase in spike response, but the main effect of these two peptides is an alteration of spike pattern. Neither has any consistent effect on the ileum. In contrast, secretin does not abolish cyclical activity, but simply reduces the intensity of cyclical spike activity to make the MMC shorter-phase $I$ is prolonged, and there are less spikes in phases II and III. Because in our studies gastric secretions were not drained, pentagastrin stimulation of acid secretion may have induced secretin release and this may have been the cause of the failure of pentagastrin (unlike CCK) to increase jejunal spike activity.

It is pertinent to ask why our analysis of type 1,2 , and 3 activity did not produce clearer changes. We have defined these types of activity (Barnett and Wingate, 1977) in terms similar to phases I-III (Code and Marlett, 1975) but there is a major difference. Phases I-III are subjective definitions of successive portions of the fasting cycle, and occur in order; the terms are inapplicable to non-cyclical activity and postprandial activity has to be described as prolonged phase II activity. Our analyser defines patterns of activity from minute to minute: 'phase II' is a mixture of all three types of activity in varying combinations. Thus, although CCK and pentagastrin abolish MMCs in some doses in some animals, the resulting persistent intermittent spike activity is not homogeneous, but contains short periods of silence (type 1), intermittent spikes (type 2), and even occasional repetitive bursts (type 3 ). Our analyser is more 'objective' but less descriptive and the results of our analysis of patterns must be taken in conjunction with the other types of analysis employed. It is clear that an ideal system of spike analysisobjective, rapid, and conclusive-does not yet exist, 
and such a system may not be possible until the phenomenology of spike response is clearer. The significance of our results is probably further obscured by the fact that we are analysing a mixture of dose-dependent changes (such as the BER) and allor-nothing changes (such as the MMC-cyclical activity is either present or absent). That being said, these studies represent the most extensive attempt to date to document the trend of change induced by these peptides.

\section{FUNCTIONAL SIGNIFICANCE}

The functional significance of this study is simply stated. On the basis of our findings, it seems highly improbable that either gastrin or CCK, contrary to previous suggestions (Weisbrodt et al., 1974; Marik and Code, 1975; Mukhopadhyay et al., 1977) are responsible, alone or in combination, for the change from fasting to postprandial motor activity. Feeding induces a rapid and sustained fall in the gastric - but not the duodenal-BER (Wingate, Thompson et al., 1977), whereas two of the three peptides studied do not alter the BER, while pentagastrin accelerates both gastric and duodenal BER. There is likewise little to suggest that secretin plays a role in the modification of motor activity. While the feeding stimulus alters the ratio of gastric to duodenal BER, pentagastrin does not, since the ratio between the two pacemaker frequencies remains relatively constant under the effects of pentagastrin;from this it is probable that the effects on gastric emptying differ from the effect of a meal. Although the change in gastric BER due to gastrin has been previously reported (Cooke et al., 1972), the effect on both pacemakers in relation to dose has not been hitherto documented.

The characteristic changes in spike activity induced by food are not reproduced by the peptides. These changes are abolition of cyclical activity (MMCs) at all levels of the small intestine, and overall increase in both jejunal and ileal spike activity (Wingate, Thompson et al., 1977. The striking difference is the failure of the ileum to respond to the peptides. The conclusion to be drawn is that the feeding response is due wholly or partly to other peptides, or neural control, or both.

If the peptides do not induce a feeding pattern, what is the purpose of the partial response? It has to be clearly understood that MMCs are propulsive (Summers et al., 1976; Bueno et al., 1975) and will propel intestinal content from pylorus to ileocaecal valve in under two hours. This type of motor activity is inappropriate for digestion; compared with fasting, digestion requires more mixing activity and less propulsive activity, and the type of change induced by CCK and gastrin achieves this to some extent. Since these are the peptides mediating exocrine digestive secretion, one may speculate that the motor effects of these hormones are to ensure that, even in the absence of other control mechanisms, digestion will take place in intestine whose motor activity is at least partially appropriate.

The persistence of fasting ileal activity in the ileum with these peptides is important in relation to current concepts of post-vagotomy diarrhoea. Allan et al. (1973) have shown that post-vagotomy diarrhoea is associated with increased colonic bile acids, known to have a cathartic effect (Mekhjian et al., 1971). The most plausible explanation is that the bile acids are swept too rapidly past the terminal ileum to allow for adequate absorption. The most powerful and propulsive gut movement is the migrating complex, and it seems reasonable to speculate that it is the persistence of distal migrating complexes after food in post-vagotomy subjects which sweeps bile acids into the colon. If vagotomy in some way disrupts the integrity of motor control and impairs the motor response to food, our study shows that raised levels of these peptides will not abolish cyclical activity in the distal intestine. Thus, the difference between the complete response to food and the incomplete response to these peptides may be of functional significance.

We are grateful to the Wellcome Trust for continued financial support, and to the Medical Research Council for a project grant.

\section{References}

Allan, J. G., Gerskowitch, V. P., and Russell, R. I. (1973). A study of the role of bile acids in the pathogenesis of postvagotomy diarrhoea (Abstract). Gut, 14, 423-424.

Barnett, T. G., and Wingate, D. L. (1977). The logical analysis of the canine electroenterogram. Journal of Physiology, 268, 14P-15P.

Bueno, L., Fioramonti, J., and Ruckebusch, Y. (1975). Rate of flow of digesta and electrical activity of the small intestine in dogs and sheep. Journal of Physiology, 249, 69-85.

Carlson, G. M., Bedi, B. S., and Code, C. F. (1971). Mechanism of propagation of intestinal interdigestive myoelectric complex. American Journal of Physiology, 222, 1027-1030.

Code, C. F., and Marlett, J. A. (1975). The interdigestive myoelectric complex of stomach and small bowel of the dog. Journal of Physiology, 246, 289-309.

Cooke, A. R., Chvasta, T. E., and Weisbrodt, N. W. (1972). Effect of pentagastrin on emptying and electrical and motor activity of the dog stomach. American Journal of Physiology, 223, 934-938.

Harvey, R. F., Oliver, J. M., Read, A. E., Ederle, A., Vantini, I., Scuro, L. A., Groarke, J. F., and Fitzgerald, O. (1977). Half-life, clearance, and endogenous secretion rates of cholecystokinin-pancreozymin in man (Abstract). Gut, 18, A982.

Hermon-Taylor, J. H., and Code, C. F. (1970). Effect of secretin on small bowel myoelectric activity of conscious healthy dogs. American Journal of Digestive Diseases, 15, 545-550. 
Lehnert, P., Stahlheber, H., Roder, O., Zoelch, M., Forell, M. M., Wünsch, E., Jaeger, E., and Martens, H. L. (1973). Bestimmung der biologischen Aktivität von synthetischem Secretin. Klinische Wochenschrift, 51, 44-46.

Marik, F., and Code, C. F. (1975). Control of the interdigestive myoelectric activity in dogs by the vagus nerves and pentagastrin. Gastroenterology, 69, 387-395.

Mekhijian, H. S., Phillips, S. F., and Hofmann, A. F. (1971). Colonic secretion of water and electrolytes induced by bile acids: perfusion studies in man. Journal of Clinical Investigation, 50, 1569-1577.

Mukhopadhyay, A. K., Thor, P. J., Copeland, E. M., Johnson, L. R., and Weisbrodt, N. W. (1977). Effect of cholecystokinin on myoelectric activity of small bowel of the dog. American Journal of Physiology, 232, E44-E47.

Oliver, J. M., and Harvey, R. F. (1977). Hormonal content of commercial preparations of cholecystokinin-pancreozymin (Abstract). Gut, 18, A982.

Summers, R. W., Helm, J., and Christensen, J. (1976). Intestinal propulsion in the dog. Its relation to food intake and the migratory myoelectric complex. Gastroenterology, 70, 753-758.
Weisbrodt, N. W., Copeland, E. M., Kearley, R. W., Moore, E. P., and Johnson, L. R. (1974). Effects of pentagastrin on electrical activity of small intestine of the dog. American Journal of Physiology, 227, 425-429.

Wingate, D. L. (1976). The eupeptide system; a general theory of gastrointestinal hormones. Lancet, 1, 529-532.

Wingate, D. L., and Barnett, T. G. (1978). Logical Analysis of the electroenterogram. American Journal of Digestive Diseases, (In press).

Wingate, D. L., Barnett, T. G., Green, W. E. R., and Armstrong-James, M. (1977). Automated high-speed analysis of gastrointestinal myoelectric activity. American Journal of Digestive Diseases, 22, 243-251.

Wingate, D. L., Thompson, H. H., Pearce, E. A., and Dand, A. (1978). Effects of exogenous cholecystokinin and pentagastrin on myoelectric activity in the small intestine of the conscious dog. Proceedings of the 6th International Symposium on Gastrointestinal Motility. (In press).

Wingate, D. L., Thompson, H. H., Pearce, E. A., and Dand, A. (1977). Quantitative analysis of the effect of feeding on canine intestinal myoelectric activity. Gastroenterology, 72, 1151 (Abstract). 